\title{
Bottomonium Hyperfine Splitting on the Lattice and in the Continuum.
}

\author{
M. Baker, ${ }^{1}$ A.A. Penin, ${ }^{1,2}$ D. Seidel, ${ }^{3}$ and N. Zerf ${ }^{1}$ \\ ${ }^{1}$ Department of Physics, University of Alberta, Edmonton, Alberta T6G 2J1, Canada \\ ${ }^{2}$ Institut für Theoretische Teilchenphysik, Karlsruhe Institute of Technology, 76128 Karlsruhe, Germany \\ ${ }^{3}$ Theoretische Physik 1, Universität Siegen, 57068 Siegen, Germany
}

\begin{abstract}
We revise the analysis of the bottomonium hyperfine splitting within the lattice nonrelativistic QCD. The Wilson coefficients of the radiatively improved lattice action are evaluated by a semianalytic approach based on the asymptotic expansion about the continuum limit. The nonrelativistic renormalization group is used to estimate the high-order radiative corrections. Our result for the $1 S$ hyperfine splitting is $M_{\Upsilon(1 S)}-M_{\eta_{b}(1 S)}=52.9 \pm 5.5 \mathrm{MeV}$. It reconciles the predictions of the continuum and lattice QCD and is in very good agreement with the most accurate experimental measurement by the Belle collaboration.
\end{abstract}

PACS numbers: $12.38 . \mathrm{Gc}, 12.38 . \mathrm{Bx}, 14.40 . \mathrm{Pq}, 14.65 . \mathrm{Fy}$

The bottomonium hyperfine splitting defined by the mass difference $E_{\mathrm{hfs}}=M_{\Upsilon(1 S)}-M_{\eta_{b}(1 S)}$ has been a subject of much controversy since the first observation of the spin-singlet $\eta_{b}$ state in radiative decays of the $\Upsilon(3 S)$ mesons by the Babar collaboration [1]. The measured value of the hyperfine splitting overshot the predictions of perturbative QCD [2] by almost a factor of two, well beyond the experimental and theoretical uncertainty

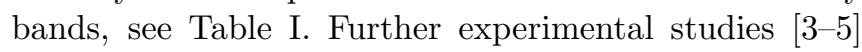
were consistent with the initial measurement, while the Belle collaboration reported a significantly lower value of the splitting with higher experimental precision [6]. On the theory side the most accurate estimates of the hyperfine splitting are obtained from lattice simulations within the effective theory of nonrelativistic QCD (NRQCD). This method is entirely based on first principles, allows for simultaneous treatment of dynamical heavy and light quarks and gives a systematic account of the longdistance nonperturbative effects of the strong interaction. The first analysis [] with fully incorporated one-loop radiative corrections [8] favored the larger value of the splitting [1]. The most recent analysis [9] includes the leading relativistic corrections and gives a lower value, which is close to the PDG average [10] but nevertheless not consistent with Ref. [2]. This might indicate a serious failure of perturbative QCD in the description of the bottomonium ground state in clear conflict with the general concept of the heavy quarkonium dynamics. Thus the current experimental and theoretical status of the bottomonium hyperfine splitting remains ambiguous and sets up one of the most interesting open problems in the QCD theory of hadrons, which yet inspired a discussion about possible signal of physics beyond the standard model [11].

In this article we revise the analysis of the radiative corrections to the lattice NRQCD action. We develop a semianalytical approach based on the asymptotic expansion about the continuum limit [12], which provides a very powerful tool for the radiative improvement of lattice NRQCD. Our result for the one-loop Wilson coefficient of the effective spin-dependent four-quark inter- action significantly differs from the result of the previous calculation [8] used in the subsequent analyses [7, 9], which leads to a sizable reduction of the lattice NRQCD prediction for the hyperfine splitting. We give an estimate of the higher order radiative corrections by evaluating the two-loop double-logarithmic terms within the nonrelativistic renormalization group approach [13, 14]. The main result of this paper is a new theoretical value for bottomonium hyperfine splitting, Eq. (11).

The idea of the NRQCD approach [15, 16] is to separate the hard modes, which require a fully relativistic analysis, from the nonrelativistic soft modes. The dynamics of the soft modes is governed by the effective nonrelativistic action given by a series in heavy quark velocity $v$, while the contribution of the hard modes is encoded in the corresponding Wilson coefficients. The nonrelativistic action can be applied in a systematic perturbative analysis of the heavy quarkonium spectrum [17 19$]$. At the same time the action may be used for lattice simulations of the heavy quarkonium states [20, 21]. The latter approach gives full control over nonperturbative long-distance effects and can be used for the description of excited states where perturbative QCD is not applicable.

The hyperfine splitting i.e. the splitting between the spin-singlet and spin-triplet states is generated by the spin-dependent part of the NRQCD Lagrangian. To or$\operatorname{der} \mathcal{O}\left(v^{4}\right)$ it reads (see e.g. 22, 23])

$$
\mathcal{L}_{\sigma}=\frac{c_{F}}{2 m_{q}} \psi^{\dagger} \boldsymbol{B} \boldsymbol{\sigma} \psi+\left(\psi \rightarrow \chi_{c}\right)+d_{\sigma} \frac{C_{F} \alpha_{s}}{m_{q}^{2}} \psi^{\dagger} \boldsymbol{\sigma} \psi \chi_{c}^{\dagger} \boldsymbol{\sigma} \chi_{c},
$$

where $\boldsymbol{B}$ is the chromomagnetic field, $m_{q}$ and $\alpha_{s}$ are the heavy quark mass and the strong coupling constant, the $S U\left(N_{c}\right)$ color group factor is $C_{F}=\left(N_{c}^{2}-1\right) /\left(2 N_{c}\right), \psi$ $\left(\chi_{c}\right)$ are the nonrelativistic Pauli spinors of quark (antiquark) field, and we have projected the four-quark interaction on the color-singlet state. The Wilson coefficients $c_{F}$ and $d_{\sigma}$ logarithmically depend on the factorization scale $\mu_{f}$ which separates the hard and the soft momen- 


\begin{tabular}{l|l}
\hline \hline \multicolumn{2}{c}{ Experiment } \\
\hline Babar, $\Upsilon(3 S)$ decays[1] & $71.4_{-3.1}^{+2.3}$ (stat) \pm 2.7 (syst) \\
Babar, $\Upsilon(2 S)$ decays [3] & $66.1_{-4.8}^{+4.9}$ (stat) \pm 2.0 (syst) \\
Belle, $h_{b}(1 P)$ decays [6] & $57.9 \pm 2.3$ \\
PDG average [10] & $62.3 \pm 3.2$ \\
\hline \multicolumn{2}{c}{ Theory } \\
\hline NRQCD, NLL [2] & $41 \pm 11(\text { th })_{-8}^{+9}\left(\delta \alpha_{s}\right)$ \\
Lattice NRQCD $\mathcal{O}\left(v^{4}\right)[7]$ & $70 \pm 9$ \\
Lattice NRQCD $\mathcal{O}\left(v^{6}\right)[9]$ & $62.8 \pm 6.7$ \\
Lattice QCD [32] & $54.0 \pm 12.4_{-0.0}^{+1.2}$ \\
Lattice NRQCD, this work & $52.9 \pm 5.5$ \\
\hline \hline
\end{tabular}

TABLE I. Results of high-precision experimental and theoretical determinations of the bottomonium hyperfine splitting in $\mathrm{MeV}$.

tum contributions. This dependence can be predicted to all orders of perturbation theory by renormalization group methods. In lattice NRQCD the natural factorization scale is given by the inverse lattice spacing $a$. The radiative improvement of the action is therefore mandatory for the correct continuum limit.

The coefficient $c_{F}$ parametrizes the quark anomalous chromomagnetic moment. It can be determined nonperturbatively by matching the lattice result for particular splittings to the physical bottomonium spectrum [7, 24]. The perturbative evaluation of the one-loop correction to $c_{F}$ [8] is in good agreement with the nonperturbative result. The Wilson coefficient of the effective four-quark interaction however can only be obtained perturbatively. It vanishes in the Born approximation and is determined by matching the one-particle irreducible quark-antiquark scattering amplitudes in QCD and NRQCD; see Fig. 1. The matching does not depend on the choice of soft kinematical variables and

becomes particulary simple when the amplitude is computed at the quark-antiquark threshold and vanishing momentum transfer. In this case the one-loop full QCD amplitude is

$$
\begin{aligned}
M_{1 \mathrm{PI}}^{\mathrm{QCD}} & =\frac{C_{F} \alpha_{s}^{2}}{m_{q}^{2}}\left[\frac{C_{A}}{2} \log \left(\frac{m_{q}}{\lambda}\right)+(\ln 2-1) T_{F}\right. \\
& \left.+\left(1-\frac{2 \pi m_{q}}{3 \lambda}\right) C_{F}\right] \psi^{\dagger} \boldsymbol{\sigma} \psi \chi_{c}^{\dagger} \boldsymbol{\sigma} \chi_{c},
\end{aligned}
$$

where $C_{A}=N_{c}, T_{F}=1 / 2$, and we introduced a small auxiliary gluon mass $\lambda$ to regulate the infrared divergence. The power enhanced $1 / \lambda$ term corresponds to the Coulomb singularity of the threshold amplitude, while the term proportional to $T_{F}$ is due to the two-gluon annihilation of the quark-antiquark pair.

On the other hand the lattice NRQCD result for the

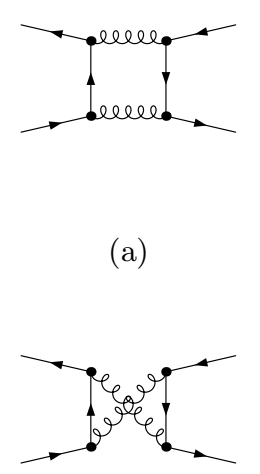

(b) (c)

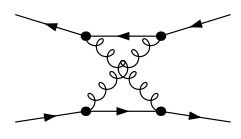

(d)

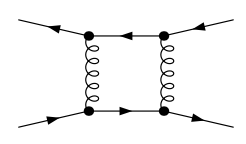

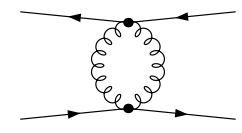

(e)

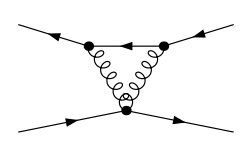

(f)
FIG. 1. One-loop Feynman diagrams contributing to the oneparticle irreducible quark-antiquark scattering amplitude in QCD [(a)-(d)] and NRQCD [(c)-(f)].

one-loop amplitude can be written as follows

$$
\begin{aligned}
M_{1 \mathrm{PI}}^{\mathrm{NRQCD}} & =\frac{C_{F} \alpha_{s}^{2}}{m_{q}^{2}}\left[-\left(\delta+\frac{1}{2} \ln (a \lambda)\right) C_{A}\right. \\
& \left.-\frac{2 \pi m_{q}}{3 \lambda} C_{F}\right] \psi^{\dagger} \boldsymbol{\sigma} \psi \chi_{c}^{\dagger} \boldsymbol{\sigma} \chi_{c}+\mathcal{O}\left(a^{2}\right),
\end{aligned}
$$

where the nonlogarithmic non-Abelian term $\delta$ depends on a particular realization of the lattice action. To match Eqs. (2) and (3) we add to the NRQCD Lagrangian the four-quark operator with coefficient

$$
d_{\sigma}=\alpha_{s}\left[\left(\delta+\frac{1}{2} L\right) C_{A}+(\ln 2-1) T_{F}+C_{F}\right],
$$

where $L=\ln \left(a m_{q}\right)$. The main problem is therefore in determination of the coefficient $\delta$. The asymptotic expansion of the lattice loop integrals about the continuum limit [12] can in principle be used to get this coefficient in a closed analytic form. Since the heavy quark mass is not a dynamical scale in NRQCD, the parameter of the expansion in our case is $a \lambda$. The idea of the method is to split the integration over the virtual momentum $l$ into the contributions of the hard region with $l \sim 1 / a$ and the soft region with $l \sim \lambda$. In the hard region the integrand is expanded in $a \lambda$ and $\lambda / l$ and reduces to the lattice tadpole integrals. In the soft region the integrand is expanded in $a l$ and $a \lambda$ and reduces to the continuum NRQCD Feynman integrals. As a result of the scale separation the hard (soft) contribution in general has spurious infrared (ultraviolet) logarithmic divergences and has to be regulated. In the total result for a given lattice loop integral the dependence on the regulator cancels out leaving the asymptotic series in $a \lambda$ which includes the logarithmic terms [cf. Eq. (3)]. We emphasize that the expansion about the continuum limit is a formal tool to get the 
NRQCD loop integrals as series in $a$ and facilitate the matching procedure, while the lattice NRQCD is a valid nonrelativistic effective theory only for $a \gg 1 / m_{q}$. Note that Eq. (3) has only a logarithmic singularity in $a$. In higher orders of the NRQCD expansion in $1 / m_{q}$ the amplitude includes also the terms with a negative power of $a$. Such $1 /\left(a m_{q}\right)^{n}$ terms are more singular in the formal continuum limit but are power suppressed with respect to Eq. (3) in the region where lattice NRQCD is applied.

Let us consider first a "naive" lattice action with no improvement for gluonic and heavy quark fields (see, e.g. [25, 26]). The gluonic field tensor of the NRQCD chromomagnetic interaction in the naive action is expressed through the commutator of the left-right symmetrized covariant lattice derivatives. In this case we obtain

$$
\delta^{\text {naive }}=-\frac{7}{3}+28 \pi^{2} b_{2}-256 \pi^{2} b_{3}=0.288972 \ldots,
$$

where the irrational constants $b_{2}=0.02401318 \ldots, b_{3}=$ $0.00158857 \ldots$ parametrize the lattice tadpole integrals and can be computed with arbitrary precision [12]. We however need the above coefficient for the improved lattice action which is used in real simulations. Analytic calculation with an improved action is not optimal since the Feynman rules in this case become extremely cumbersome. We bypass this problem by using a semianalytic approach. Indeed the difference between the Wilson coefficients for the improved and naive lattice actions $\Delta \delta$ remains finite in the limit $\lambda \rightarrow 0$ and can be directly obtained by numerical evaluation of the corresponding lattice loop integrals with sufficiently small $\lambda$ (a finite infrared regulator is necessary for the stability of numerical integration). For the numerical implementation of the improved lattice action Feynman rules we use HiPPy/HPsrc code 27]. However in contrast to the standard implementation the color space reduction is performed analytically with the help of the program COLOR [28] before the numerical integration is done by the CUBA integrator library [29]. This greatly reduces the runtime and allows for a separate treatment of the contributions of independent color group structures which have different infrared properties, $c f$. Eq. (3). The whole process of the calculation is fully automated. In the case of the HPQCD action 7] we get $\Delta \delta=-0.1444(28)$ corresponding to

$$
\delta=0.1446(28) .
$$

Note that since $d_{\sigma}=0$ in the Born approximation, we have to perform neither the strong coupling constant renormalization nor the lattice tadpole improvement. We made a few cross-checks of the calculation. For the naive action the numerical evaluation agrees with the gaugeinvariant analytic result of the asymptotic expansion for small values of $\lambda$. The logarithmic part of $d_{\sigma}$ is in agreement with the renormalization group analysis. The nonrelativistic renormalization group predicts the all-order

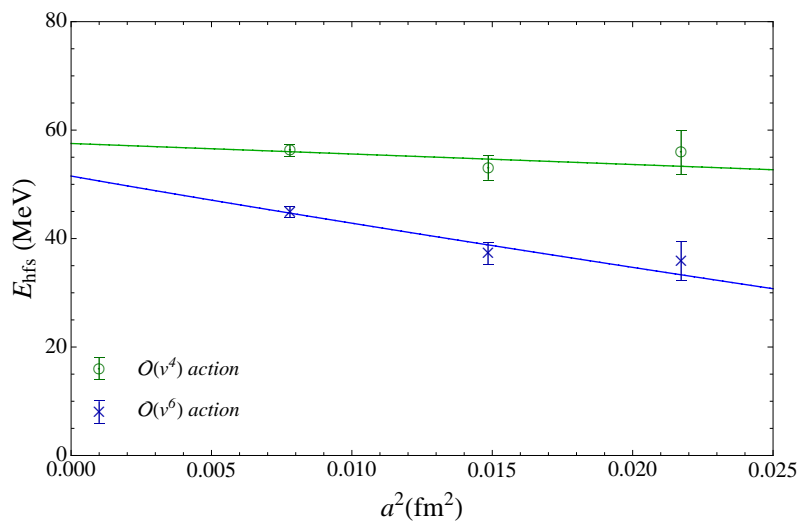

FIG. 2. The result of the lattice simulation of the bottomonium hyperfine splitting with $\mathcal{O}\left(v^{4}\right)$ action [7] and $\mathcal{O}\left(v^{6}\right)$ action [9]. The error bars are explained in the text. The solid lines correspond to the central values of the constrained fit.

dependence of the Wilson coefficients on $\mu_{f}[13,14]$. In the leading logarithmic approximation they read

$$
d_{\sigma}^{L L}=\frac{C_{A}}{\beta_{0}-2 C_{A}}\left(z^{-2 C_{A}}-z^{-\beta_{0}}\right), \quad c_{F}^{L L}=z^{-C_{A}},
$$

where $\beta_{0}=11 C_{A} / 3-4 T_{F} n_{l} / 3$ is the one-loop QCD $\beta$ function, $n_{l}=4$ is the number of light flavors, and $z=\left(\alpha_{s}\left(\mu_{f}\right) / \alpha_{s}\left(m_{q}\right)\right)^{1 / \beta_{0}}$. In lattice NRQCD the factorization scale should be identified with inverse lattice spacing $\mu_{f} \sim 1 / a$. By reexpanding the leading logarithmic result we obtain

$$
\begin{aligned}
\frac{d_{\sigma}^{L L}}{\pi} & =\frac{\alpha_{s}}{\pi} \frac{C_{A}}{2} L-\left(\frac{\alpha_{s}}{\pi}\right)^{2} \frac{\left(\beta_{0}+C_{A}\right) C_{A}}{4} L^{2}+\ldots, \\
c_{F}^{L L} & =1-\frac{\alpha_{s}}{\pi} \frac{C_{A}}{2} L+\left(\frac{\alpha_{s}}{\pi}\right)^{2} \frac{\left(\beta_{0}+C_{A}\right) C_{A}}{8} L^{2}+\ldots,
\end{aligned}
$$

in agreement with Eq. (4).

Let us now compare our result with the previous calculation [8]. In this paper a different basis of the four-quark operators is used and the Wilson coefficient $d_{\sigma} / \alpha_{s}$ should be identified with the linear combination $\frac{9}{8}\left(d_{1}-d_{2}\right)$ (see [30] for the consistent analytical expressions). We find that the nonannihilation constant term of the QCD amplitude in [8] is smaller than the one in Eq. (2) by a factor of 3 . The comparison of the NRQCD part of the result is not straightforward since in [8, 30] it has been evaluated numerically for three different lattice spacings keeping the full dependence on $m_{q}$. This dependence includes power suppressed terms as well as the linear term from the lattice cutoff of the Coulomb pinch contribution. For the lattice spacing used in real simulations $a \sim 1 /\left(v m_{q}\right)$ the power suppressed terms are of the same magnitude as the generic one-loop relativistic corrections and are beyond the accuracy of our analysis. On the contrary the lattice artifacts are numerically significant. The linear term associated with the Coulomb pinch can be estimated by cutting the corresponding continuum NRQCD 


\begin{tabular}{l|c|c}
\hline \hline & $\mathcal{O}\left(v^{4}\right)$ action & $\mathcal{O}\left(v^{6}\right)$ action \\
\hline Discretization error & 2.6 & 3.1 \\
Relativistic corrections & 6.0 & 1.8 \\
Radiative corrections & 4.8 & 4.3 \\
\hline$E_{\mathrm{hfs}}$ & 57.5 & 51.5 \\
\hline \hline
\end{tabular}

TABLE II. The central value and the error budget for the lattice NRQCD determination of the bottomonium hyperfine splitting with $\mathcal{O}\left(v^{4}\right)$ action [7] and $\mathcal{O}\left(v^{6}\right)$ lattice action [9] in $\mathrm{MeV}$.

one-loop integral at the scale $\pi / a$. This gives an additional contribution to Eq. (4),

$$
-\nu \frac{8}{3} \frac{C_{F} \alpha_{s}}{\pi} a m_{q} \approx-0.94 \alpha_{s} a m_{q}
$$

where the factor $\nu=0.831 \ldots$ adjusts the analytical result for the integral over spherical momentum domain to the integral over the Brillouin zone. The numerical result of Ref. [8] suggests a significantly larger negative coefficient of about -1.8 . Moreover in the threshold region the multiple Coulomb gluon exchange contributions are not parametrically suppressed and the modification of the Coulomb bound state dynamics on the finite lattice is not accounted for by the one-loop analysis. It may change the numerical coefficient of the linear term and generates all-order contributions in $\alpha_{s} a m_{q}$. This means that (i) the one-loop matching does not remove the linear lattice artifact at $\mathcal{O}\left(\alpha_{s}\right)$ and (ii) one cannot use the finite lattice spacing $a \sim 1 /\left(\alpha_{s} m_{q}\right)$ as a Wilsonian cutoff for NRQCD as it was done in [8, 30]. Thus all the lattice artifacts should be removed nonperturbatively by numerical extrapolation of the lattice data to $a=0[\overline{7}, 9]$.

Now we are in a position to apply our result to the analysis of the hyperfine splitting. The contribution of the four-quark interaction to $E_{\mathrm{hfs}}$ reads

$$
\Delta E_{\mathrm{hfs}}=-d_{\sigma} \frac{4 C_{F} \alpha_{s}}{m_{q}^{2}}|\psi(0)|^{2},
$$

where $\psi(0)$ is the wave function of the quarkonium ground state at the origin. Equation (10) should be added to the result of the lattice simulation with the one-loop Wilson coefficient $c_{F}$ and no four-quark contribution included. Such a result is available for the $\mathcal{O}\left(v^{4}\right)$ action [7] and for the $\mathcal{O}\left(v^{6}\right)$ action [9]. For the numerical analysis of Eq. (10) we use the nonperturbative lattice result for $\psi(0)$ 7]. To make our analysis self consistent we adopt the value of the bottom quark mass $m_{b}$ and the value of the strong coupling constant $\alpha_{V}$ renormalized in the static potential scheme at the scale $\pi / a$ from Ref. [7]. The numerical result for the hyperfine splitting is presented in Fig. 2 as a function of $a^{2}$ for three different lattice spacings and two different lattice actions. The error bars of each point include the statistical er- ror and the uncertainty in the value of the lattice spacing from [7, 9] as well as the high-order $a$-dependent radiative corrections that are estimated by the size of the double-logarithmic two-loop terms in Eq. (8). The use of relatively large values of the lattice spacing $a \sim 1 /\left(v_{b}\right)$ ensures the suppression of the unphysical $1 /\left(a m_{b}\right)^{n}$ contributions, which become important at $a \sim 1 / m_{b}[7,30]$. At the same time it results in sizable lattice artifacts, which cannot be removed by finite order matching due to the all-order character of the Coulomb binding effects. To minimize this effect the result is numerically extrapolated to $a=0$ [7, 9]. The extrapolation below $a \sim 1 / m_{b}$ in this case is justified since the numerical effect of the $1 /\left(a m_{b}\right)^{n}$ terms on the data points is small. To perform the extrapolation we use a constrained fit of the data points 31] by a polynomial in $a$ with vanishing linear term. The inclusion of the linear and $1 /\left(a m_{b}\right)^{n}$ terms in the fit is discussed below. To estimate the coefficients of the higher order terms in the lattice spacing we represent the result of the fit as $1+(\Lambda a)^{2}+\mathcal{O}\left(a^{3}\right)$, where $\Lambda$ is the mass scale characterizing the approach of the lattice approximation to the continuum limit. The priors for the coefficients of the $a^{n}$ terms with $n>2$ in the constrained fit are then given by the intervals $\pm \Lambda^{n}$. Numerically we get $\Lambda \approx 360 \mathrm{MeV}$ for the $\mathcal{O}\left(v^{4}\right)$ and $\Lambda \approx 790 \mathrm{MeV}$ for the $\mathcal{O}\left(v^{6}\right)$ case. Because of a slower approach to the continuum limit the extrapolation error for $\mathcal{O}\left(v^{6}\right)$ action turns out to be larger. This may be related to the fact that the contribution of the operators of higher dimension is more sensitive to the ultraviolet momentum region. Therefore the currently unknown $\mathcal{O}\left(\alpha_{s} v^{6}\right)$ matching corrections in this approximation can be substantial. We checked that the inclusion of the $1 / a^{n}$ terms with the priors $\frac{\alpha_{s}}{\pi}\left(\frac{\pi}{m_{b}}\right)^{n}$ into the constrained fit changes the result within the extrapolation error intervals.

In general the Coulomb binding effects give rise to a linear dependence of the lattice data on $a$ which can be roughly estimated by the one-loop result (9). A more refined estimate can be obtained by including the linear term $c_{l} \alpha_{s} a m_{q}$ into the fit of the lattice data. For the prior $\left|c_{l}\right|<1$ the constrained fit gives $c_{l} \approx-0.25$ for both actions, which is two times smaller than the oneloop estimate $c_{l} \approx-0.5$ corresponding to Eq. (9). At the same time the extracted value of the hyperfine splitting is increased within the extrapolation error interval by approximately $2.5 \mathrm{MeV}$.

The total error budget of our estimate is given in Table III. Besides the discretization errors discussed above it includes the uncertainty due to high-order relativistic and radiative corrections. For a conservative estimate of the radiative corrections we take the value of the doublelogarithmic two-loop terms at the soft factorization scale $\mu_{f} \approx \alpha_{s} m_{b}$ dictated by the bound state dynamics. In Table II this uncertainty is combined with the numerical error in the one-loop coefficient $c_{F}$ [9]. Our estimate of the relativistic corrections for the $\mathcal{O}\left(v^{4}\right)$ action is based 
on the difference between the $\mathcal{O}\left(v^{4}\right)$ and $\mathcal{O}\left(v^{6}\right)$ results in the continuum limit. For the $\mathcal{O}\left(v^{6}\right)$ action we multiply this uncertainty by $\alpha_{s}$ evaluated at the soft renormalization scale to take into account the previously discussed missing matching corrections. The larger discretization uncertainty balances the smaller relativistic corrections in the $\mathcal{O}\left(v^{6}\right)$ case and both actions provide comparable total errors. Since the structure of the relativistic corrections and the behavior of the results at finite lattice spacing are significantly different for the two actions, we consider the corresponding uncertainties as uncorrelated and take the weighted average of the results as the best estimate. At the same time the uncertainty due to the high-order purely radiative corrections is treated as correlated between the two actions. Our final result for the hyperfine splitting reads

$$
E_{\mathrm{hfs}}=52.9 \pm 5.5 \mathrm{MeV} \text {. }
$$

We now can compare our estimates to the available theoretical and experimental results in Table I. Our result for both $\mathcal{O}\left(v^{4}\right)$ and $\mathcal{O}\left(v^{6}\right)$ actions (Table II) are below the ones of the previous lattice NRQCD analysis [7, 9] by approximately $12 \mathrm{MeV}$. About $5 \mathrm{MeV}$ of the difference is due to the error in the one-loop QCD amplitude calculation [8]. The remaining discrepancy is related to the different procedure of extrapolation to $a=0$. The analysis [7, 9] implies that the one-loop matching [8] removes the linear artifact form the lattice data. However, as it was pointed out above, the one-loop calculation can only be used for a rough estimate of the linear term due to the all-order character of the Coulomb binding effects. We therefore determine the corresponding coefficient by a constrained fit of the lattice data with the prior set by the one-loop result. Moreover the numerical result [8] suggests a significantly larger value of the linear term than what follows from our analytic calculation and from the fit of the lattice data, which leads to a sizable difference of the extrapolation results.

With the new value of the four-quark Wilson coefficient the lattice NRQCD prediction (11) agrees within the error bars with the next-to-leading logarithmic (NLL) perturbative QCD result [2]. Its central value practically coincides with that of the full lattice QCD simulation [32], though the uncertainty of the latter is significantly larger. This may indicate that the matching of the lattice NRQCD to full QCD is now done properly. On the experimental side our result strongly favors the value obtained by the Belle collaboration, which has the lowest reported uncertainty. Thus we have reconciled the theoretical predictions of the lattice and continuum QCD as well as the most accurate experimental data.

The work was supported in part by NSERC. The work of A.P. is supported by the Perimeter Institute for Theoretical Physics. Research at the Perimeter Institute is supported by the Government of Canada through Industry Canada and by the Province of Ontario through the Ministry of Research and Innovation.

[1] B. Aubert et al. [BaBar Collaboration], Phys. Rev. Lett. 101, 071801 (2008) [Erratum-ibid. 102, 029901 (2009)].

[2] B. A. Kniehl, A. A. Penin, A. Pineda, V. A. Smirnov and M. Steinhauser, Phys. Rev. Lett. 92, 242001 (2004).

[3] B. Aubert et al. [BaBar Collaboration], Phys. Rev. Lett. 103, 161801 (2009).

[4] G. Bonvicini et al. [CLEO Collaboration], Phys. Rev. D 81, 031104 (2010).

[5] S. Dobbs, Z. Metreveli, K. K. Seth, A. Tomaradze and T. Xiao, Phys. Rev. Lett. 109, 082001 (2012).

[6] R. Mizuk et al. [Belle Collaboration], Phys. Rev. Lett. 109, 232002 (2012).

[7] R. J. Dowdall et al. [HPQCD Collaboration], Phys. Rev. D 85, 054509 (2012).

[8] T. C. Hammant, A. G. Hart, G. M. von Hippel, R. R. Horgan and C. J. Monahan, Phys. Rev. Lett. 107, 112002 (2011).

[9] R. J. Dowdall et al. [HPQCD Collaboration], Phys. Rev. D 89, no. 3, 031502 (2014).

[10] J. Beringer et al. [Particle Data Group Collaboration], Phys. Rev. D 86, 010001 (2012).

[11] F. Domingo, U. Ellwanger and M. A. Sanchis-Lozano, Phys. Rev. Lett. 103, 111802 (2009).

[12] T. Becher and K. Melnikov, Phys. Rev. D 66, 074508 (2002).

[13] A. A. Penin, A. Pineda, V. A. Smirnov and M. Steinhauser, Phys. Lett. B 593, 124 (2004).

[14] A. A. Penin, A. Pineda, V. A. Smirnov and M. Steinhauser, Nucl. Phys. B 699, 183 (2004).

[15] W. E. Caswell and G. P. Lepage, Phys. Lett. B 167, 437 (1986).

[16] G. T. Bodwin, E. Braaten and G. P. Lepage, Phys. Rev. D 51, 1125 (1995).

[17] N. Brambilla, A. Pineda, J. Soto and A. Vairo, Phys. Lett. B 470, 215 (1999).

[18] B. A. Kniehl, A. A. Penin, V. A. Smirnov and M. Steinhauser, Nucl. Phys. B 635, 357 (2002).

[19] A. A. Penin and M. Steinhauser, Phys. Lett. B 538, 335 (2002).

[20] B. A. Thacker and G. P. Lepage, Phys. Rev. D 43, 196 (1991).

[21] G. P. Lepage, L. Magnea, C. Nakhleh, U. Magnea and K. Hornbostel, Phys. Rev. D 46, 4052 (1992).

[22] A. Pineda and J. Soto, Phys. Rev. D 58, 114011 (1998).

[23] A. Pineda and J. Soto, Phys. Rev. D 59, 016005 (1999).

[24] S. Meinel, Phys. Rev. D 82, 114502 (2010).

[25] H. J. Rothe, "Lattice Gauge Theories: An Introduction", World Sci. Lect. Notes Phys. 59, 1 (1997).

[26] E. Eichten and B. R. Hill, Phys. Lett. B 240, 193 (1990).

[27] A. Hart, G. M. von Hippel, R. R. Horgan and E. H. Muller, Comput. Phys. Commun. 180, 2698 (2009).

[28] T. van Ritbergen, A. N. Schellekens and J. A. M. Vermaseren, Int. J. Mod. Phys. A 14 (1999) 41.

[29] T. Hahn, Comput. Phys. Commun. 168 (2005) 78.

[30] T. C. Hammant, A. G. Hart, G. M. von Hippel, R. R. Horgan and C. J. Monahan, Phys. Rev. D 88, 
014505 (2013).

[31] G. P. Lepage, B. Clark, C. T. H. Davies, K. Hornbostel, P. B. Mackenzie, C. Morningstar and H. Trottier, Nucl. Phys. Proc. Suppl. 106, 12 (2002).
[32] T. Burch, C. DeTar, M. Di Pierro, A. X. El-Khadra, E. D. Freeland, S. Gottlieb, A. S. Kronfeld and L. Levkova et al., Phys. Rev. D 81, 034508 (2010). 